Article

\title{
Semi-Empirical Capacity Fading Model for SoH Estimation of Li-Ion Batteries
}

\author{
Preetpal Singh ${ }^{1}\left(\mathbb{D}\right.$, Che Chen ${ }^{2}$, Cher Ming Tan ${ }^{1,2,3,4,5, *}$ and Shyh-Chin Huang ${ }^{1,3}$ \\ 1 Center for Reliability Engineering, Ming Chi University of Technology, New Taipei City 24301, Taiwan \\ 2 Centre for Reliability Science and Technology, Chang Gung University, Wenhua 1st Road, GuishanDist., \\ Taoyuan City 33302, Taiwan \\ 3 Department of Mechanical Engineering, Ming Chi University of Technology, New Taipei City 24301, Taiwan \\ 4 Department of Electronics Engineering, Chang Gung University, Wenhua 1st Rd., Guishan Dist., \\ Taoyuan City 33302, Taiwan \\ 5 Department of Urology, Chang Gung Memorial Hospital, Guishan, Taoyuan City 33302, Taiwan \\ * Correspondence: cherming@ieee.org; Tel.: +886-3-2118800-5801
}

Received: 24 April 2019; Accepted: 24 July 2019; Published: 26 July 2019

\begin{abstract}
A fast and accurate capacity estimation method for lithium-ion batteries is developed. This method applies our developed semi-empirical model to a discharge curve of a lithium-ion battery for the determination of its maximum stored charge capacity after each discharge cycle. This model provides an accurate state-of-health $(\mathrm{SoH})$ estimation with a difference of less than $2.22 \%$ when compared with the electrochemistry-based electrical (ECBE) SoH calculation. The model parameters derived from a lithium-ion battery can also be applied to other cells in the same pack with less than $2.5 \%$ difference from the complex ECBE model, showing the extendibility of the model. The parameters ( $\mathrm{k} 1, \mathrm{k} 2$, and $\mathrm{k} 3$ ) calculated in the work can also be used to study the changes in battery internal structure, such as capacity losses at normal conditions, as well as cycling at high temperatures. The time for estimation after each discharge cycle is only $5 \mathrm{~s}$, making it is suitable for on-line in-situ estimation.
\end{abstract}

Keywords: semi-empirical capacity fading model; ECBE model; Li-ion batteries; robust and accurate

\section{Introduction}

With the growing awareness of the energy crisis and environmental pollution, electric vehicles using electricity as an energy source are gradually becoming the focus of attention. The energy source usually consists of a rechargeable lithium-ion battery ( $\mathrm{LiB})$.

Unlike other types of batteries, LiB offers higher energy efficiency. However, the health of a LiB and its cost of replacement have been the problems for consumers in electric vehicle applications, and the development of an accurate and quick, preferably in-situ health monitoring method is receiving significant research attention in the field of LiBs [1]. As the discharge characteristics of a LiB vary with the composition of the electrochemistry in the battery, and the state-of-health $(\mathrm{SoH})$ of a LiB is strongly affected by its composition as well as ambient environmental factors, together with variation in its usage conditions, the estimation of the LiB health can be challenging. This state-of-health is a "measurement" that reflects the general health condition of a battery in term of its ability to deliver maximum stored charge as compared to its fresh state. It depends on factors such as its maximum stored charge, internal resistance, voltage, and self-discharge. Theoretically, the $\mathrm{SoH}$ of a fresh battery is $100 \%$ and will decrease over time due to its usage [2]. State-of-health is an indicator to describe the health status of a battery, and it can reflect the performance and aging of the battery. In terms of electrical quantity, $\mathrm{SoH}$ is an indicator of the remaining full battery capacity. SoH is generally defined 
as the ratio of the maximum releasable capacity of a battery to the rated capacity of the battery when it is fresh.

The two most important health conditions of a LiB are its maximum charge capacity, which is represented by $\mathrm{SoH}$, and its status of safety, which is represented by state-of-safety (SoS). SoS represents the condition when the battery is in danger to use in the vehicles, and it can be estimated by several means, such as its thermal runaway, current, voltage, state-of-charge (SoC), and $\mathrm{SoH}$ [3]. As $\mathrm{SoH}$ degrades over charge-discharge cycles, accurate estimation of its SoC can be affected if the $\mathrm{SoH}$ is not taken into account. This is because SoC is defined as the percentage of available stored charge with respect to the full capacity of the battery. This full capacity of the battery is sometimes its rated capacity, but more likely its current (i.e., at the latest charge-discharge cycle) capacity and this leads to an ambiguity that renders confusion and differences in the reported SoC values. During the lithiation process (charging), metal oxides break apart from the cathode side, which produces Li ions that bond with the anode side material with the addition of electrons. The opposite process where Li-ion travels back and bond with the metal oxide on the cathode side is called the de-lithiation (discharging) process. The effectiveness of the cathode material is determined based on the number of $\mathrm{Li}$ ions produced during the lithiation process, and the effectiveness of the anode material is verified based on the number of Li ions which migrate from cathode and bond with the anode material. This, in turn, also decides the SoC and SoH of the batteries [4]. To estimate the maximum distance before the LiBs need to be charged again requires the knowledge of the road conditions ahead and an accurate SoC, and this can be represented by the state-of-energy (SoE) $[5,6]$. SoE indicates the remaining energy, instead of charge, that can be used from the battery, which describes a more precise state or condition of the battery. The state-of-discharge $(\mathrm{SoD})$ is the percentage of the discharged capacity of a battery which is fully charged before discharging and is related to SoC as [7].

$$
\mathrm{SoD}=1-\mathrm{SoC} \text {. }
$$

Uncertainties in capacity estimation can cause the LiBs to be over-discharged will accelerate the degradation of LiBs. These uncertainties can also cause the users to be over-conservative and increase the number of charge-discharge cycles of $\mathrm{LiBs}$ and accelerate the degradation of $\mathrm{LiB}$ as well. In this work, we focus on the estimation of SoH as it forms the basis for accurate SoS, SoC, and SoE estimations.

There are many methods reported for measuring battery capacity. Among the electrical methods, the common methods are Coulomb counting [8], open-circuit voltage (OCV) [9], extended Kalman filter (EKF), and the neural network [10]. The Coulomb counting is simple and easy to implement, but it is also easy to have accumulated error due to measurement error/uncertainty of the factors, such as current, temperature, etc. OCV is another common method, but it can only be applied in the open circuit condition. In addition, the flat portion of the OCV curve with SoC can lead to significantly inaccurate estimation. EKF is a modification of the Kalman filter to ease its implementation, and thus it offers a computationally efficient option for runtime SOC evaluation for on-board vehicles. However, the error-prone calculations of the Jacobian matrix, as well as the lack of robustness due to the linearization process and instability of the filters, are the shortcomings of the EKF method. The neural network has adaptability and a self-learning ability, but it needs large numbers of experimental data to train the network to acquire good prediction results. As battery cells are degraded, training is again required, making the training resources expensive. Many physical models based on the dynamics of the battery have been purposed recently. Xia et al. [11] and Jiang et al. [12] developed an equivalent circuit based model to estimate battery dynamics. Huang et al. [13] defined a new variable for the variation of the potential to perform data regression on $\mathrm{SoC}$ and SoH estimation. The electrochemistry-based electrical (ECBE) model that was developed in our previous work is an electrical model developed based on the first principle of electrochemistry $[9,14]$. This model can compute the internal parameters of a cell, such as $Q_{m}, m_{1}, m_{2}$, and, $k$, from its discharge curve and the change in these parameters, can be used to describe the degradation (including SoH degradation) of an $\mathrm{LiB}$. Here $\mathrm{Q}_{\mathrm{m}}$ is the maximum charge storage of a battery, and it is an important factor for calculating the SoH. The total amount of 
charge extracted by the external circuit is not equal to the maximum charge stored due to the non-zero cut-off voltage for safety consideration and the electrochemical impedance inside a battery caused by cycle aging. $\mathrm{m}_{1}$ expresses the effectiveness of a cathode in storing Li-ions and its decrease indicates a degradation in the ability of the cathode to effectively accept Li-ions. $\mathrm{m}_{2}$ expresses the effectiveness of the graphite anode to providing its stored Li-ions, and its decrease indicates degradation in the ability of the anode to effectively deliver Li-ions. $\mathrm{k}$ is the rate of charge transfer from electrodes to the electrolyte, and it can be used to measure microscopic polarization properties, and its reduction represents degradation at the electrodes' interface that affects the kinetics of electrochemical reactions at the electrodes [15].

While the ECBE model is accurate and comprehensive, the required computation time to obtain the values of the model parameters is excessive and renders its unsuitability for in-situ SoH estimation. In this work, a semi-empirical capacity fading model [16] is investigated to determine the SoH of the cells under cycle aging test. This model can describe the cell's degradation behavior due to discharge rate, temperature, and cycle numbers. We use the ECBE model as a reference to assess the accuracy of the semi-empirical model in this work. In other words, the ECBE model has a more complex structure and requires longer computation time, rendering it difficult to implement practically. On the contrary, the semi-empirical model is presented by a closed-form formula, and thus, the required computation time is much reduced as compared to the ECBE model. Another advantage of the semi-empirical method is the ease of obtaining parameters, such as $\mathrm{k} 1, \mathrm{k} 2$, and $\mathrm{k} 3$, which describes the internal state of the battery by implementing a single formula. The parameters $(k 1, k 2$, and $k 3)$ calculated in the work can also be used to study the changes in battery internal structure, such as capacity losses at normal conditions, as well as cycling at high temperatures. In this work, we use the ECBE model as a reference.

Several empirical prediction models have been proposed in recent years. R.B. Wright et al. developed a calendar and cycle life model, which account for the time, temperature, and SoC of batteries [17]. J. Wang established the cycle-life models that followed capacity loss related to time or charge to estimate the capacity fade of LiFePO4 [18]. The mechanical-fatigue method adopts damage-accumulation relationships to evaluate the capacity fade [19]. And some studies use a coupled model to achieve life-prediction [20,21]. However, these prediction models consider several factors, such as temperature, depth of discharge (DOD), current rate, etc., rendering their applications to describe capacity fade complicated as temperature, $\mathrm{DOD}$, and current are independent variables. In contrast, the semi-empirical model chosen in this work eliminates the dependency of these factors, making it more practical to the estimation of SoH. In this work, we simplify this semi-empirical model so that it can be presented by a closed-form equation, reducing the required computation time significantly as compared to ECBE and other models. The parameters of this equation can also be obtained easily, and these parameters describe the internal states of the battery.

The present paper is organized as follows: The experimental settings and approach are given in Section 2, and an introduction of the principle and architecture of the semi-empirical capacity fading model is discussed in the subsequent Section. The differences in the estimation results with ECBE are presented from our experimental results. Conclusions and future works are given in the last Section.

\section{Materials and Methods}

We tested eight batteries [22] using Bio-Logic BCS-815 (Samsung SDI CO.,LTD. Yongin, South Korea), which is an 8-channels tester and support 15 A per channel. Bio-Logic BCS-815 can record the experimental parameters of time, voltage, current, surface temperature, and charge counting. The chemical type of the batteries used in this experiment was Nickel-Manganese-Cobalt Oxide (NMC), in which the cathode is composed of Lithium-Nickel-Manganese-Cobalt Oxide ( $\mathrm{Li}\left[\mathrm{Ni}, \mathrm{Mn}, \mathrm{Co}^{\mathrm{O}} \mathrm{O}_{2}\right)$ and the anode is composed of Graphite. We performed charge and discharge of the batteries at room temperature $\left(25^{\circ} \mathrm{C}\right)$. The discharge cycle data was recorded every $1 \mathrm{~s}$. The specifications of the batteries used here are shown in Table 1 as obtained from the manufacturer. Due to the limited number of test facilities at present, we could only test 8 batteries during this experiment. 
Table 1. Samsung 18,650 battery specification provided by the manufacturer [22].

\begin{tabular}{ll}
\hline Battery Characteristics & Description \\
\hline Type Cylindrical & \\
Chemical system & $\mathrm{NMC}$ \\
Chemical component of the cathode & $\mathrm{Li}[\mathrm{Ni}, \mathrm{Mn}, \mathrm{Co}] \mathrm{O}_{2}$ \\
Chemical component of the anode & Graphite \\
Nominal voltage & $3.62 \mathrm{~V}$ \\
Typical capacity & $2150 \mathrm{mAh}$ \\
Charge cut-off voltage & $4.2 \mathrm{~V}$ \\
Discharge cut-off voltage & $2.75 \mathrm{~V}$ \\
Dimensions(mm) & $18.4 \times 65$ \\
Approx. weight & $44.5 \mathrm{~g}$ \\
\hline
\end{tabular}

Eight batteries were divided into 4 groups consisting of 2 batteries each, and each group had a difference of 50 cycles of charging-discharging. We implemented this process to observe the effect of varying cycle number on the performance of the batteries. In this experiment, we used the Constant current-Constant Voltage (CC-CV) method to charge the batteries. 1 A constant current (as recommended from the battery's datasheet) was used to charge the batteries to its cut-off voltage, then the charging was done with a constant voltage until the current dropped to $100 \mathrm{~mA}$. When the battery was fully charged, we implemented $1 \mathrm{C}$-rate (typical capacity $=2.15 \mathrm{Ah}$ ) to discharge the batteries until the cut-off voltage of $2.75 \mathrm{~V}$ was reached. Figure 1 shows a typical terminal voltage curve during charge and discharge of the batteries used in this work. The initial discharge voltage was around $3.9 \mathrm{~V}$ instead of $4.2 \mathrm{~V}$ due to the voltage drop caused by battery internal resistance [23] when the voltage drops to $2.75 \mathrm{~V}$ that means the end of discharge operation.

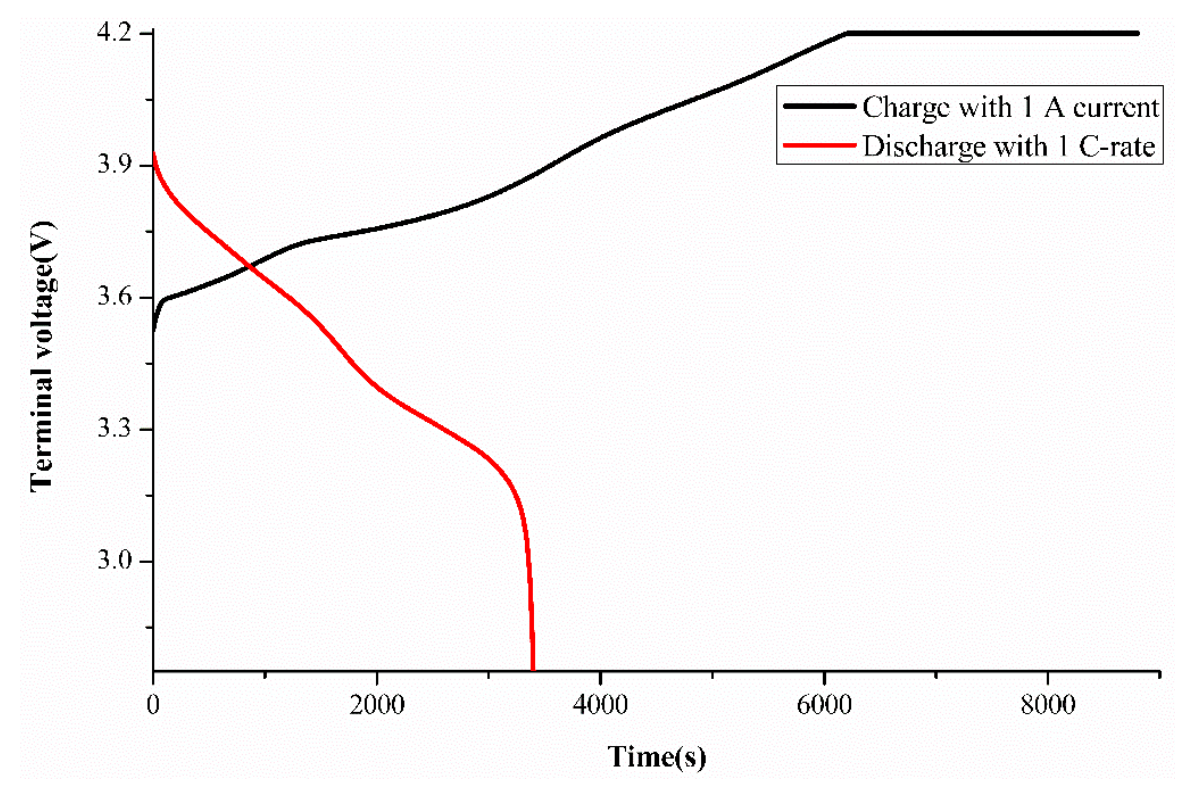

Figure 1. Variation of terminal voltage recorded during the charge-discharge period.

\subsection{Semi-Empirical Capacity Fading Model}

After the acquisition of the charge-discharge data of a cell, we analyzed the discharge curve using the ECBE model to calculate the maximum capacity of the cell $\left(\mathrm{Q}_{\mathrm{m}}\right)$. Experimental SoH was computed for the batteries tested for various cycles using Equation $(2)[8,16,24]$ using the maximum capacity of the batteries after the first cycle $\left(\mathrm{Q}_{\max }(\right.$ fresh $\left.)\right)$ and after aging $\left(\mathrm{Q}_{\max }(\right.$ aged $\left.)\right)$.

$$
\mathrm{SoH}=\frac{Q_{\max (\text { aged })}}{Q_{\max (\text { fresh })}}
$$


The semi-empirical model used in this work is described by Equation (3) [16]. Parameters (k1, k2, k3) in the model can be extracted by substituting SoH obtained using Equation (2) from 3 different cycles into Equation (3). The extracted parameters were used to calculate the $\mathrm{SoH}$ over the entire battery life and denoted as estimated $\mathrm{SoH}$ in this work.

$$
\mathrm{SoH}=1-\left(\frac{1}{2} \mathrm{k}_{1} N^{2}+\mathrm{k}_{2} N\right)-\frac{\mathrm{k}_{3}}{Q_{\max (\text { fresh })}} i,
$$

where $\mathrm{k} 1, \mathrm{k} 2$, and $\mathrm{k} 3$ are the coefficients that depend on battery operation conditions. $\mathrm{k} 1$ represents the capacity loss that increases rapidly at high ambient temperature, and $\mathrm{k} 2$ is a factor to account for capacity loss at normal temperature. $\mathrm{k} 3$ is a parameter that represents the capacity loss caused by the discharge rate. $\mathrm{N}$ represents the number of charge-discharge cycles the battery experienced at the time of this $\mathrm{SoH}$ calculation, and $i$ is the discharging current.

\subsection{ECBE Model}

We introduced the principle of ECBE model in our previous work $[9,14]$. We developed the ECBE model based on the principle of electrochemistry and then converted the corresponding partial differential equations into a circuit model. We used simulated annealing to obtain an approximated global minimum and the non-linear regression method. Later we employed the Levenberg-Marquart fitting algorithm (LMA) to provide an accurate estimate of the local minima.

\subsection{Experimentation}

Figure 2 depicts our experimental procedure.



Figure 2. Flowchart for difference estimation between experimental and estimated $\mathrm{SoH}$.

\section{Results and Discussion}

Calculation of experimental SoH was performed using the Qm value obtained from the ECBE model for each cell tested for a different number of cycles, and the results are shown in Table 2. The SoH drops as the cycle number increases as expected. Two decimals point were used inconsistently with 
the accuracy of the SoH values obtained using the ECBE model as verified in our previous work (its accuracy is better than $99.6 \%$ ) [9].

Table 2. The state-of-health (SoH) obtained from the electrochemistry-based electrical (ECBE) model for each cycle and cell.

\begin{tabular}{lcccccccc}
\hline Cycle $\backslash$ Cell & A1 & A2 & A3 & A4 & A5 & A6 & A7 & A8 \\
\hline 1 & 100 & 100 & 100 & 100 & 100 & 100 & 100 & 100 \\
100 & 95.93 & 97.16 & 97.42 & 97.48 & 98.41 & 97.93 & 98.11 & 97 \\
200 & 95.35 & 96.65 & 96.23 & 95.94 & 96.21 & 96.19 & 96.91 & 96.14 \\
300 & 93.65 & 94.42 & 94.92 & 94.27 & 94.8 & 94.95 & 95.06 & 94.03 \\
400 & 91.64 & 92.08 & 92.72 & 92.15 & 93.43 & 92.86 & 93.57 & 91.26 \\
500 & 90.12 & 89.83 & 89.66 & 89.81 & 90.83 & 90.08 & 90.97 & 88.76 \\
550 & - & - & 89.17 & 88.64 & 89.55 & 88.34 & 88.27 & 87 \\
600 & - & - & - & - & 87.53 & 86.8 & 85.86 & 84.95 \\
650 & - & - & - & - & - & - & 82.93 & 82.57 \\
\hline
\end{tabular}

An interesting observation from our experiments is that batteries tested for a similar number of cycles under the same stress conditions show a difference in SoH drop, which signifies variation among the batteries. This could be due to the different imbalance in the internal state for an individual battery [25]. This renders it difficult to estimate single battery life, but users need to know the degradation of each of their batteries, instead of the entire population of batteries. Furthermore, a large number of battery samples is needed to estimate the population of the batteries, and this can be costly. Furthermore, one would expect a large standard deviation of the batteries degradation rate as electrochemistry degradation cannot be tuned as precisely as electronic components, in general.

The computed SoH values were used to obtain the $\mathrm{k} 1, \mathrm{k} 2$, and $\mathrm{k} 3$ values using the above-mentioned semi-empirical capacity fading model with three different cycle numbers. We arbitrarily chose data of the $40^{\text {th }}, 150^{\text {th }}$, and $250^{\text {th }}$ cycle for all the batteries which are marked by a green circle in Figure 3 . The computed values are shown in Table 3, and again, it is observed that the k values vary among the batteries from the same set tested under the same conditions for an equal number of cycles. This is in correlation with the $\mathrm{SoH}$ results which could be due to the difference in capacity degradation [26,27]. In other words, assessing the $\mathrm{SoH}$ of batteries tested from the same batch under the same working conditions for companies' quality and reliability check can be difficult. It is also interesting to note that a few of the parameters have negative values. The negative $k 1$ and $k 2$ values were observed for the batteries with higher SoH which shows that there is an improvement in battery health. Some of the defects arising during the battery lifetime can enhance the battery lifetime due to improvement in the overall speed at which Li ion travels as published by Hong et. al. [28]. Researchers believe that an electrode's lithium transport properties are fundamentally changed due to particle defects and this can enable lithium ions to penetrate inside the cathode in multiple directions. The reactive surface area increases in this process and more efficient exchange of $\mathrm{Li}$ ions is possible between the two electrodes [29]. However, more negative values were observed for $\mathrm{k} 3$ but the values are very close to 0 and thus can be ignored. These negative values could also be a result of a measurement error, thus we used two batteries at varying discharged cycle numbers and found that the negative values arise only for two batteries except for parameter $\mathrm{k} 3$. This shows the efficiency of the model used in this work.

We use the computed $\mathrm{k}$ values for each cell to determine the $\mathrm{SoH}$ of the cell over the number of tested cycles, as shown in Figure 3, where Cell A2 is used for demonstration. Green circles indicate the points (the range of the half-cycle period) which were used to find the k values in the last step. The values of $k 1$ and $k 2$ were severely affected by the ambient temperature, while $k 3$ was affected by the discharge current. The operating conditions of this experiment were room temperature and 1 C-rate discharge current, so we can observe that the difference between k-values of all cells is not large, but there is still a gap between individual samples. 
Table 3. The parameters of $k 1, k 2$, and $k 3$ are calculated from the SoH formula.

\begin{tabular}{lccc}
\hline Cell $\backslash$ K-Value & $\mathbf{k 1}$ & $\mathbf{k 2}$ & $\mathbf{k 3}$ \\
\hline A1 & $-3.28 \times 10^{-7}$ & $1.779 \times 10^{-4}$ & 0.013339 \\
A2 & $1.73 \times 10^{-7}$ & $1.21 \times 10^{-4}$ & 0.012944 \\
A3 & $9.71 \times 10^{-7}$ & $-3.64 \times 10^{-5}$ & 0.0241156 \\
A4 & $1.24 \times 10^{-7}$ & $1.87 \times 10^{-4}$ & -0.0081339 \\
A5 & $7.39 \times 10^{-8}$ & $1.81 \times 10^{-4}$ & -0.00796576 \\
A6 & $2.63 \times 10^{-7}$ & $9.98 \times 10^{-5}$ & 0.00652144 \\
A7 & $1.97 \times 10^{-7}$ & $5.15 \times 10^{-5}$ & 0.025597 \\
A8 & $2.38 \times 10^{-7}$ & $2.19 \times 10^{-4}$ & -0.0092757 \\
\hline
\end{tabular}

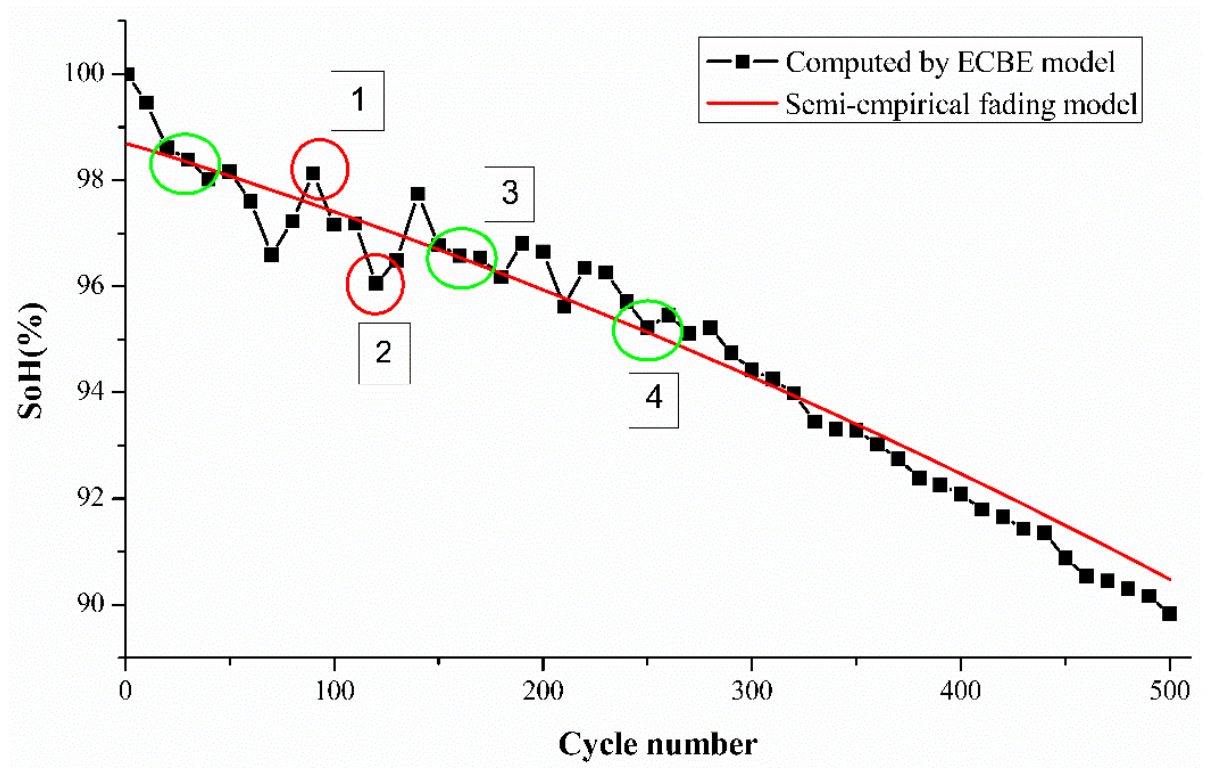

Figure 3. State-of-health $(\mathrm{SoH})$ estimation curve for cell A2 to compare the difference between experimental $\mathrm{SoH}$ and estimated $\mathrm{SoH}$.

From Figure 3, it is evident that the differences between the experimental $\mathrm{SoH}$ and estimated $\mathrm{SoH}$ vary over their lifetime for the tested cells. The estimation differences are higher for cycle numbers from 1 to 60 . The rapid degradation of SoH from 1 to 60 cycles is likely to be attributed to the transformation of the hexagonal structure to a cubic spinel structure of the electrode which occurs via an intermediate phase as observed previously by Tan et al. [15].

To further validate our ECBE model results, we have also added the Coulomb counting result in Figure 4. However, it was mentioned in the introduction that there are many disadvantages to employing the Coulomb counting method, as mentioned by researchers. Coulomb counting employs an open-loop estimator, which generates larger cumulative errors. It also does not take a change in the battery's capacity into account as the battery ages [30]. Other disadvantages of the open-loop are its sensitivity to the current sensor precision and uncertainty of initial SoC [31]. It also shows an increase in charge at certain cycles, as observed during the ECBE charge estimation results. The higher value of Qm over Qd is expected, as explained in Leng et al.'s work [15]. A very interesting point is observed from Figure 4 regarding the charge increase at various aging cycles. This is an unusual phenomenon observed in our results, and we would like to investigate it further by performing a charge cycle-dependent test for batteries. This will be our future work, and we would hope to share our findings from our test with the scientific community very soon. 


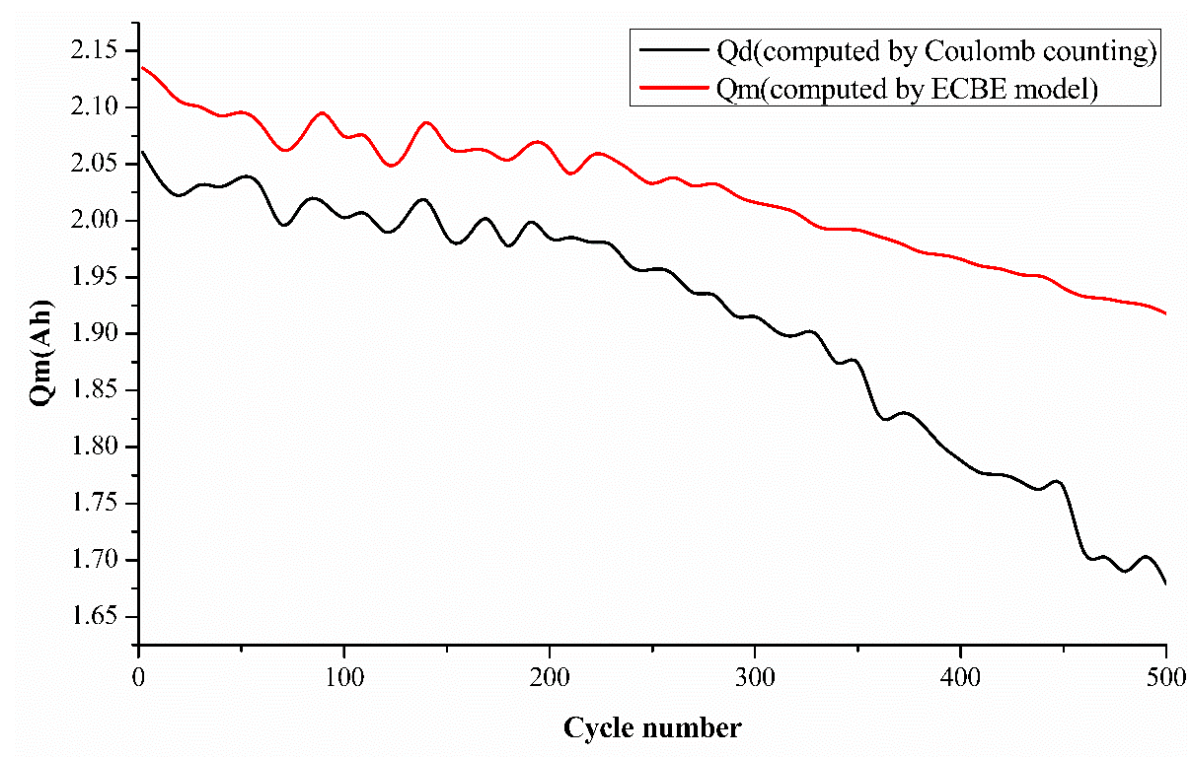

Figure 4. Charge evaluation for a Li-ion battery using the electrochemistry-based electrical (ECBE) model and Coulomb counting method.

To probe further into the differences in the estimation to investigate the usefulness of the model for SoH estimation, a comparison of the differences between the estimated and experimental SoH over the battery lifetime was performed.

\subsection{Comparison of Estimation Difference for the Case of an Individual Cell}

To verify the ability of the semi-empirical fading model for estimating the $\mathrm{SoH}$ of batteries over the number of cycles tested as shown previously, the percentage difference (which will be termed as difference hereafter) was calculated for each cell between the experimental and estimated $\mathrm{SoH}$ and the results are shown in Figure 5.

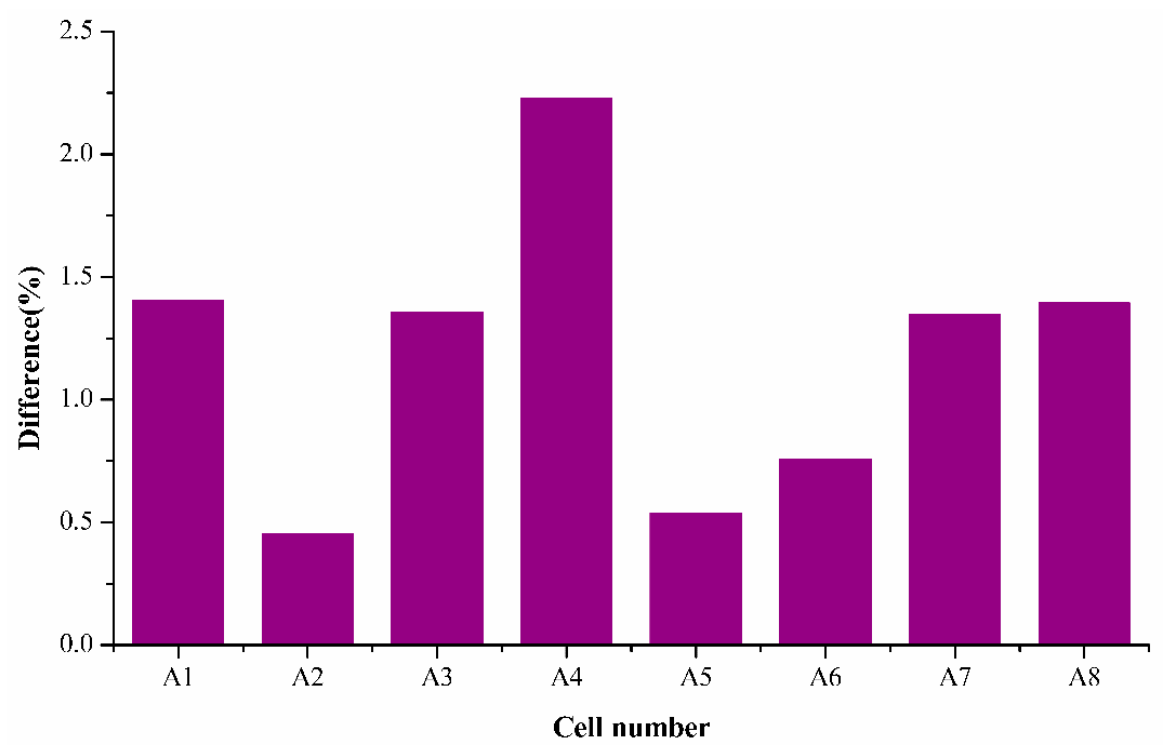

Figure 5. Comparison result between the estimated and experimental results for the ECBE model verification for each cell after the completion of the test (Cell A1, A2 are tested for 500 cycles, A3, A4 are tested for 550 cycles, A5, A6 are tested for 600 cycles, and A7, A8 are tested for 650 cycles).

It is noticed that the average mean difference was around $1.2 \%$, which is an acceptable difference according to literature. The highest percentage difference was found to be $2.22 \%$ of $\mathrm{A} 4$, and the 
remaining samples were all within the acceptable range of $1.5 \%$ which demonstrates the capability of the semi-empirical fading model in accurately estimating the decrease of $\mathrm{SoH}$ for all the cells tested for whole cycles ranging. It is also interesting to note that difference for cells A2, A5, and A6 are very low (around $0.5 \%$ ), which represents the model's effectiveness in determining accurate $\mathrm{SoH}$.

After verifying the ability of the semi-empirical fading model of a LiB cell, we examined the ability of the extracted $\mathrm{k}$ values to estimate the $\mathrm{SoH}$ of other cells from the same batch. To verify this, average, $\mathrm{k}$ values obtained in our previous steps were calculated, as shown in Table 4, and they were used to estimate the $\mathrm{SoH}$ of the batteries over the number of cycles tested.

Table 4. Averaged K-values.

\begin{tabular}{llll}
\hline & k1 & k2 & k3 \\
\hline Averaged K-value & $2.14 \times 10^{-7}$ & $1.25 \times 10^{-4}$ & 0.007143 \\
\hline
\end{tabular}

The average values in Table 4 were used to estimate the $\mathrm{SoH}$ of the batteries over the number of cycles tested, and the comparison results are shown in Figure 6. Cell A2 is used to demonstrate the comparison. It is again observed that the estimation difference is higher for cycle numbers from 1 to 60 , as noticed in our previous results. However, the mean difference is still within the range of around $1 \%$ in our case.
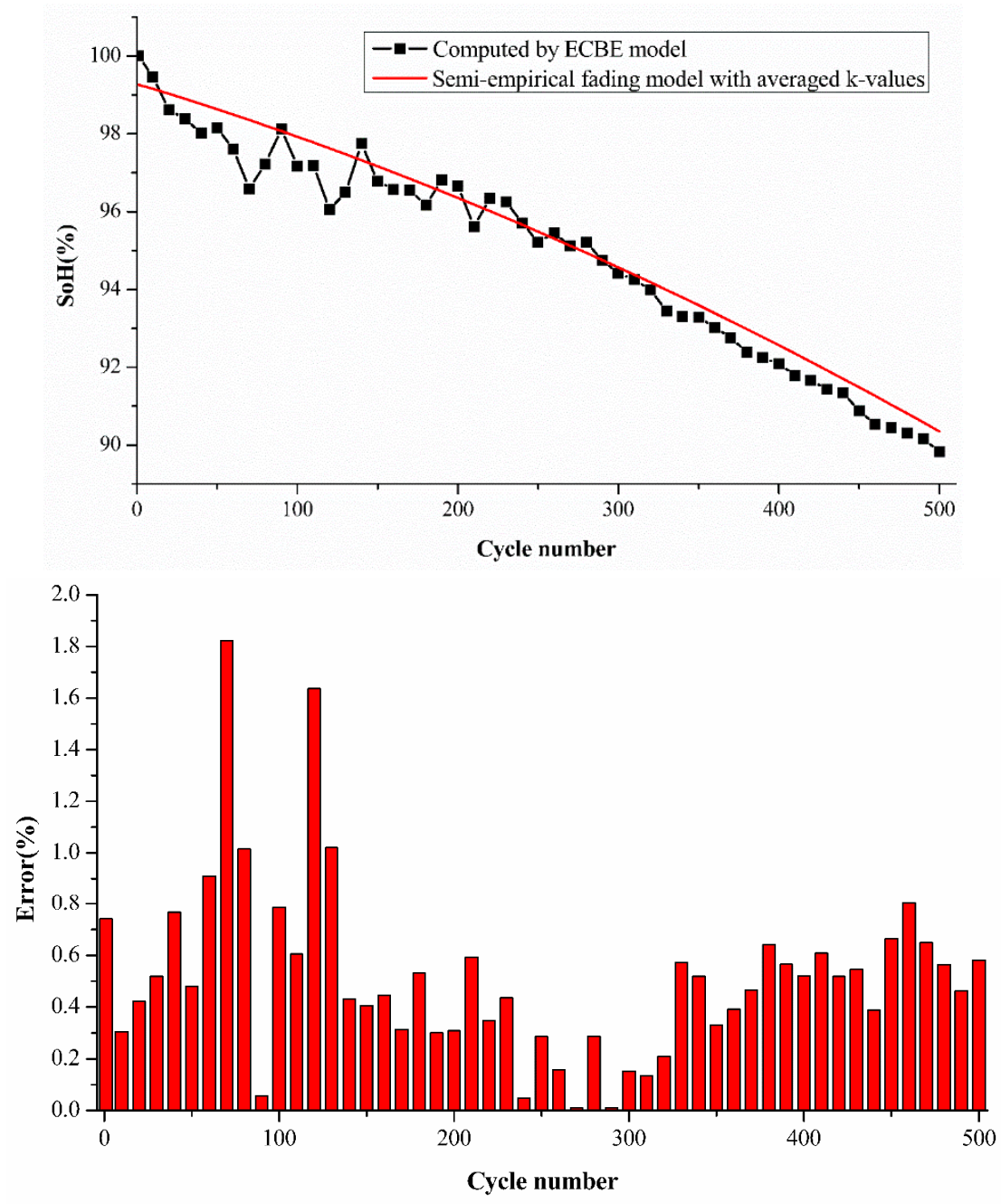

Figure 6. Difference comparison between the battery fade model using average parameters and the ECBE model for cell A2. 
Figure 6 shows the differences between the estimated $\mathrm{SoH}$ and the experimental SoH for cell A2 using the average $\mathrm{k}$ values, and the differences are in acceptable limits at each cycle number, up to 500 cycles. The initial and final part are prone to larger differences, but the actual battery degradation can still be accurately estimated from the trend of semi-empirical fading model (red line). Figure 7 shows the average percentage difference for individual cells when estimated using the average $\mathrm{k}$ values, and it is observed that the capacity fading model fitting is in good correlation with the experimental $\mathrm{SoH}$ data obtained with the ECBE model with the average $\mathrm{k}$ values. The mean difference percentage value is less than $1 \%$, which is even better than the estimations of an individual cell. This shows the robustness of the semi-empirical fading model in estimating SoH for a batch of cells over their respective number of cycles.



Figure 7. Mean difference comparison result using averaged K-value of each cell tested after the completion of the test at the respective charge-discharge cycles mentioned in Figure 5.

\subsection{Comparison of Estimation Difference for Batch of Cells}

Apart from estimating an individual cell's SoH using their respective extracted $\mathrm{k}$ values and average $\mathrm{k}$ values, it is also interesting to observe the potential of the semi-empirical fading model to estimate $\mathrm{SoH}$ of different cells using the extracted $\mathrm{k}$ values from one cell. This can show the practicality of the semi-empirical fading model for use in estimating $\mathrm{SoH}$ for a large number of cells in a set by knowing the $\mathrm{k}$ values of only one cell's parameters. Thus, $\mathrm{k}$ values obtained from an individual cell were used to estimate the $\mathrm{SoH}$ of other cells, and the difference percentage was calculated for all the cells. The results for the cell difference estimation are presented in Figure 8 below.

Figure 8 shows the results of the percentage difference for $\mathrm{SoH}$ estimation when $\mathrm{k}$ values from one cell are used for various cell's SoH estimation tested from the same batch. The various colors represent the cell number whose k values are used for SoH estimation in Figure 8. For example, the bar represented in the yellow circle represents the difference percentage of cell 1 when its SoH is estimated using the $\mathrm{k}$ values extracted from cell 2.

The results of the percentage difference are generally less than $2.5 \%$ for all the cells, as seen from Figure 8 The difference for cell 7 and 8 which were tested for 650 cycles is in an acceptable range using the estimation using the values obtained from 250 cycles, half of the total cycle numbers estimated. This demonstrates that the simplified model can predict or estimate the $\mathrm{SoH}$ with good accuracy using the half-life cell data. Thus, the proposed model can save time in testing and provides rapid SoH estimation with acceptable accuracy. 




Figure 8. The Histogram of estimation of the mean difference calculated from each cell by their K-value. The "K-values from A1" means the (k1, k2, k3) obtained from the cell A1.

To verify the sensitivity and accuracy of the present semi-empirical model when different charge cycles are used, we estimated average error for the samples using maximum and minimum peak values. As an example, we chose cycles, as shown by value 1,3, and 4 once and value 2,3 , and 4 to observe the sensitivity of the model, as shown in Figure 3. A similar observation was done for other samples as well, and the error difference is shown in the Table below.

It is clearly observed from Table 5 that the difference in errors estimated using upper and lower peaks is less than or around 1\% showing the robustness of the model. Cells were chosen from different sets which show that the model is suitable for charge estimation at the different life cycle of batteries, which is an important factor while estimating charge of Li-ion batteries.

Table 5. Difference in average error estimation while choosing upper and lower peaks to observe the semi-empirical model sensitivity.

\begin{tabular}{lll}
\hline Cell & Upper Peak $(\mathbf{1}, \mathbf{3}, \mathbf{4})$ & Lower Peak $(\mathbf{2}, \mathbf{3}, \mathbf{4})$ \\
\hline A1 & $1.6162 \%$ & $1.1857 \%$ \\
A3 & $1.6446 \%$ & $2.6735 \%$ \\
A6 & $1.807 \%$ & $1.5575 \%$ \\
\hline
\end{tabular}

In fact, since the fluctuation occurs in the first 250 cycles, and battery, in general, will have a lifetime longer than 250 cycles, we can also perform our computation of $\mathrm{k} 1, \mathrm{k} 2$, and $\mathrm{k} 3$ using the ECBE model from 250 cycles onward. In so doing, the average error in charge estimation is less than $1 \%$, which is a lot more accurate, as shown in Table 6 below. 
Table 6. Average error during charge estimation of Li-ion batteries' charge at different cycles using cycle number higher than 250 .

\begin{tabular}{cc}
\hline Cell & Averaged Error \\
\hline A1 & $0.46 \%$ \\
A3 & $0.53 \%$ \\
A5 & $0.81 \%$ \\
A7 & $0.82 \%$ \\
\hline
\end{tabular}

\subsection{Model Effectiveness for Other Types of Battery}

To demonstrate the effectiveness of the model for other types of batteries, a simplified model was used to estimate SoH for two Lithium Cobalt Oxide (LCO) batteries tested for 220 cycles under ambient conditions similar to the experimental set up shown previously for NMC batteries. The LCO sample used in this experiment was a MOLICEL ICR-18650M (MOLICEL $囚$, Taipei, Taiwan) with a typical capacity of 2.8 $\mathrm{Ah}$ [32], and its electrodes were composed of $\mathrm{LiCoO}_{2}$ and a graphite anode. The charging current set in the experiment was $1 \mathrm{~A}$, and discharge was $1 \mathrm{C}$-rate $(=2.8 \mathrm{~A})$. Due to the limitation of time and instruments, only two samples were tested as the purpose was simply to demonstrate its effectiveness in principle. The estimation difference is shown in Table 7 , and the average differences are $1.77 \%$ and $1.03 \%$, respectively. The difference is found to be less than 2 percent. The SoH comparison between the ECBE and semi-empirical model is shown in Figure 9, which includes the discharge curve of the LCO battery as an inset.

Table 7. The estimation difference of Lithium Cobalt Oxide (LCO) batteries.

\begin{tabular}{ll}
\hline Sample Number & Difference from ECBE \\
\hline A1 & $1.77 \%$ \\
A2 & $1.03 \%$ \\
\hline
\end{tabular}



Figure 9. SoH estimation curve for Lithium Cobalt Oxide (LCO) cell to compare the difference between experimental $\mathrm{SoH}$ and estimated SoH. Inset graph shows the discharge curve of LCO batteries.

The above fitting results demonstrate that the semi-empirical fading model used in this work can rapidly estimate SoH of batteries accurately. It is also observed that the difference values are always less than $2 \%$ whether the SoH estimation is done using its own extracted $\mathrm{k}$ values or another cell's $\mathrm{k}$ values. Another important observation is that this method provides accurate SoH estimation even when the first 60 cycles are included in SoH estimation, where rapid capacity decrease is observed. 
This rapid capacity decrement increases the estimation difference and can be further reduced if the first 60 cycles are not included in the SoH estimation. However, we have included the first 60 cycles in this work for SoH estimation to show the accuracy of the simplified model in SoH estimation. It will be very interesting to check the credential of this method when batteries degrade to even lower $\mathrm{SoH}$ values, and it will be one of our future works. In short, our model provides a path for SoH estimation of several cells in a pack by just the extraction of $k$ values for a single cell in that pack.

\section{Conclusions}

The requirement for an accurate online estimation of a LiB battery capacity is increasing. We applied the ECBE model and semi-empirical model in this work to estimate SoH. The ECBE model was used for initial $\mathrm{SoH}$ calculation to determine the parameters in our semi-empirical model, which can then be used for SoH estimation of cells for their subsequent charge-discharge cycles.

A reliable and simple approach to the estimation of the battery cycle life is provided by the semi-empirical fading model. The SoH estimations for two different types of $\mathrm{Li}$-ion batteries were verified in this paper. The accuracy of the semi-empirical fading model was demonstrated through batteries tested for varying cycle numbers. The maximum difference percentage for $\mathrm{SoH}$ estimation was found to be around $2.22 \%$ when the $\mathrm{k}$ values obtained from an individual cell were used for its own SoH estimation, especially the maximum estimated difference using the averaged $\mathrm{k}$ values was only $1.2 \%$. The model gives almost less than $2.5 \%$ difference in $\mathrm{SOH}$ estimation when $\mathrm{k}$ values of one cell were used to estimate another cell's SoH, making it practical for SoH estimation, especially where the estimation time after each discharge cycle is only $5 \mathrm{~s}$ using a personal computer with the capability of 8 GB RAM and Intel Core i5 processor.

Author Contributions: C.M.T. conceived and designed the research; P.S., C.C., and C.M.T. analyzed the testing data; P.S., S.-C.H., C.C., and C.M.T. wrote the paper.

Funding: The authors would like to acknowledge the support by Taiwan's Ministry of Science and Technology under the Grant Numbers MOST 107-2632-E-131-002 and Chang Gung University under Grant number QZRPD123.

Acknowledgments: The authors would like to acknowledge the support of Centre of Reliability Science and Technologies Lab, Chang Gung University and Center on Reliability Engineering, the Ming Chi University of Technology for providing valuable equipment and support for smooth conduct of experiments.

Conflicts of Interest: The authors declare no conflict of interest.

\section{References}

1. Berecibar, M.; Gandiaga, I.; Villarreal, I.; Omar, N.; VanMierlo, J.; Van DenBossche, P. Critical review of state of health estimation methods of Li-ion batteries for real applications. Renew. Sustain. Energy Rev. 2016, 56, 572-587. [CrossRef]

2. Wang, T.; Zhu, C.; Pei, L.; Lu, R.; Xu, B. The State of Arts and Development Trend of SOH Estimation for Lithium-Ion Batteries. In Proceedings of the 2013 IEEE Vehicle Power and Propulsion Conference (VPPC), Beijing, China, 15-18 October 2013; pp. 1-6.

3. Cabrera-Castillo, E.; Niedermeier, F.; Jossen, A. Calculation of the state of safety (SOS) for lithium ion batteries. J. Power Sources 2016, 324, 509-520. [CrossRef]

4. Casimir, A.; Zhang, H.; Ogoke, O.; Amine, J.C.; Lu, J.; Wu, G. Silicon-based anodes for lithium-ion batteries: Effectiveness of materials synthesis and electrode preparation. Nano Energy 2016, 27, 359-376. [CrossRef]

5. Liu, X.; Wu, J.; Zhang, C.; Chen, Z. A method for state of energy estimation of lithium-ion batteries at dynamic currents and temperatures. J. Power Sources 2014, 270, 151-157. [CrossRef]

6. Mamadou, K.; Delaille, A.; Lemaire-Potteau, E.; Bultel, Y. The State-of-Energy: A New Criterion for the Energetic Performances Evaluation of Electrochemical Storage Devices. ECS Trans. 2010, 25, 105-112.

7. Moo, C.S.; Ng, K.S.; Chen, Y.P.; Hsieh, Y.C. State-of-Charge Estimation with Open-Circuit-Voltage for Lead-Acid Batteries. In Proceedings of the 2007 Power Conversion Conference-Nagoya, Nagoya, Japan, 2-5 April 2007; pp. 758-762. 
8. Ng, K.S.; Moo, C.-S.; Chen, Y.P.; Hsieh, Y.C. Enhanced coulomb counting method for estimating state-of-charge and state-of-health of lithium-ion batteries. Appl. Energy 2009, 86, 1506-1511. [CrossRef]

9. Leng, F.; Tan, C.M.; Yazami, R.; Le, M.D. A practical framework of electrical based online state-of-charge estimation of lithium ion batteries. J. Power Sources 2014, 255, 423-430. [CrossRef]

10. Charkhgard, M.; Farrokhi, M. State-of-Charge Estimation for Lithium-Ion Batteries Using Neural Networks and EKF. IEEE Trans. Ind. Electron. 2010, 57, 4178-4187. [CrossRef]

11. Xia, B.; Zhao, X.; deCallafon, R.; Garnier, H.; Nguyen, T.; Mi, C. Accurate Lithium-ion battery parameter estimation with continuous-time system identification methods. Appl. Energy 2016, 179, 426-436. [CrossRef]

12. Jiang, B.; Dai, H.; Wei, X.; Zhu, L.; Sun, Z.; Jiang, B.; Dai, H.; Wei, X.; Zhu, L.; Sun, Z. Online Reliable Peak Charge/Discharge Power Estimation of Series-Connected Lithium-Ion Battery Packs. Energies 2017, 10, 390. [CrossRef]

13. Huang, S.C.; Tseng, K.H.; Liang, J.W.; Chang, C.L.; Pecht, M.G. An online SOC and SOH estimation model for lithium-ion batteries. Energies 2017, 10, 512. [CrossRef]

14. Leng, F.; Tan, C.M.; Pecht, M. Effect of Temperature on the Aging rate of Li Ion Battery Operating above Room Temperature. Sci. Rep. 2015, 5, 12967. [CrossRef]

15. Leng, F.; Wei, Z.; Tan, C.M.; Yazami, R. Hierarchical degradation processes in lithium-ion batteries during ageing. Electrochim. Acta 2017, 256, 52-62. [CrossRef]

16. Liu, Z.; Tan, C.; Leng, F. A reliability-based design concept for lithium-ion battery pack in electric vehicles. Reliab. Eng. Syst. Saf. 2015, 134, 169-177. [CrossRef]

17. Wright, R.; Motloch, C.; Belt, J.; Christophersen, J.; Ho, C.; Richardson, R.; Bloom, I.; Jones, S.; Battaglia, V.; Henriksen, G.; et al. Calendar- and Cycle-life studies of advanced technology development program generation 1 lithium-ion batteries. J. Power Sources 2002, 110, 445-470. [CrossRef]

18. Wang, J.; Liu, P.; Hicks-Garner, J.; Sherman, E.; Soukiazian, S.; Verbrugge, M.; Tataria, H.; Musser, J.; Finamore, P. Cycle-life model for graphite-LiFePO 4 cells. J. Power Sources 2011, 196, 3942-3948. [CrossRef]

19. Safari, M.; Morcrette, M.; Teyssot, A.; Delacourt, C. Life-Prediction Methods for Lithium-Ion Batteries Derived from a Fatigue Approach. J. Electrochem. Soc. 2010, 157, A713. [CrossRef]

20. Li, Z.; Lu, L.; Ouyang, M.; Xiao, Y. Modeling the capacity degradation of $\mathrm{LiFePO}_{4} /$ graphite batteries based on stress coupling analysis. J. Power Sources 2011, 196, 9757-9766. [CrossRef]

21. Ecker, M.; Gerschler, J.B.; Vogel, J.; Käbitz, S.; Hust, F.; Dechent, P.; Sauer, D.U. Development of a lifetime prediction model for lithium-ion batteries based on extended accelerated aging test data. J. Power Sources 2012, 215, 248-257. [CrossRef]

22. SPECIFICATION OF PRODUCT for Lithium-ion Rechargeable Cell. 2010. Available online: http://gamma. spb.ru/media/pdf/liion-lipolymer-lifepo4-akkumulyatory/ICR18650-22P.pdf (accessed on 12 October 2017).

23. Saha, B.; Goebel, K. Modeling Li-ion Battery Capacity Depletion in a Particle Filtering Framework. In Proceedings of the Annual Conference of the Prognostics and Health Management Society, San Diego, CA, USA, 27 September-1 October 2009.

24. Shahriari, M.; Farrokhi, M. Online State-of-Health Estimation of VRLA Batteries Using State of Charge. IEEE Trans. Ind. Electron. 2013, 60, 191-202. [CrossRef]

25. Manenti, A.; Abba, A.; Geraci, A.; Savaresi, S. A New Cell Balancing Architecture for Li-ion Battery Packs Based on Cell Redundancy. IFAC Proc. Vol. 2011, 44, 12150-12155. [CrossRef]

26. Zheng, Y.; Ouyang, M.; Lu, L.; Li, J. Understanding aging mechanisms in lithium-ion battery packs: From cell capacity loss to pack capacity evolution. J. Power Sources 2015, 278, 287-295. [CrossRef]

27. Baumhöfer, T.; Brühl, M.; Rothgang, S.; Sauer, D.U. Production caused variation in capacity aging trend and correlation to initial cell performance. J. Power Sources 2014, 247, 332-338. [CrossRef]

28. Hong, L.; Li, L.; Chen-Wiegart, Y.K.; Wang, J.; Xiang, K.; Gan, L.; Li, W.; Meng, F.; Wang, F.; Wang, J.; et al. Two-dimensional lithium diffusion behavior and probable hybrid phase transformation kinetics in olivine lithium iron phosphate. Nat. Commun. 2017, 8, 1194. [CrossRef]

29. Yu, Y.X. Can all nitrogen-doped defects improve the performance of graphene anode materials for lithium-ion batteries? Phys. Chem. Chem. Phys. 2013, 15, 16819. [CrossRef]

30. Hansen, T.; Wang, C.J. Support vector based battery state of charge estimator. J. Power Sources 2005, 141, 351-358. [CrossRef] 
31. Zou, Y.; Hu, X.; Ma, H.; Li, S.E. Combined State of Charge and State of Health estimation over lithium-ion battery cell cycle lifespan for electric vehicles. J. Power Sources 2015, 273, 793-803. [CrossRef]

32. Facility, T. MODEL ICR18650M Lithium-ion Rechargeable Battery. Available online: http://www. houseofbatteries.com/documents/ICR18650M.pdf (accessed on 18 July 2017).

(C) 2019 by the authors. Licensee MDPI, Basel, Switzerland. This article is an open access article distributed under the terms and conditions of the Creative Commons Attribution (CC BY) license (http://creativecommons.org/licenses/by/4.0/). 